\title{
A disintegrin-metalloproteinase prevents amyloid plaque formation and hippocampal defects in an Alzheimer disease mouse model
}

\author{
Rolf Postina, ${ }^{1}$ Anja Schroeder, ${ }^{1}$ Ilse Dewachter, ${ }^{2}$ Juergen Bohl, ${ }^{3}$ Ulrich Schmitt, ${ }^{4}$ Elzbieta Kojro, ${ }^{1}$ \\ Claudia Prinzen, ${ }^{1}$ Kristina Endres, ${ }^{1}$ Christoph Hiemke, ${ }^{4}$ Manfred Blessing, ${ }^{5,6}$ Pascaline Flamez, ${ }^{7}$ \\ Antoine Dequenne, ${ }^{7}$ Emile Godaux, ${ }^{7}$ Fred van Leuven, ${ }^{2}$ and Falk Fahrenholz ${ }^{1}$ \\ ${ }^{1}$ Institute of Biochemistry, University of Mainz, Mainz, Germany. ${ }^{2}$ Experimental Genetics Group, Katholieke Universiteit Leuven, Leuven, Belgium. \\ ${ }^{3}$ Department of Neuropathology, ${ }^{4}$ Department of Psychiatry, and 5Department of Pathophysiology, University of Mainz, Mainz, Germany. \\ ${ }^{6}$ Centre for Biotechnology and Biomedicine (BBZ), Department of Veterinary Medicine, University of Leipzig, Leipzig, Germany. \\ ${ }^{7}$ Laboratory of Neuroscience, University of Mons-Hainaut, Mons-Hainaut, Belgium.
}

\begin{abstract}
Alzheimer disease (AD) is characterized by excessive deposition of amyloid $\beta$-peptides (A $\beta$ peptides) in the brain. In the nonamyloidogenic pathway, the amyloid precursor protein (APP) is cleaved by the $\alpha$-secretase within the A $\beta$ peptide sequence. Proteinases of the ADAM family (a disintegrin and metalloproteinase) are the main candidates as physiologically relevant $\alpha$-secretases, but early lethality of knockout animals prevented a detailed analysis in neuronal cells. To overcome this restriction, we have generated transgenic mice that overexpress either ADAM10 or a catalytically inactive ADAM10 mutant. In this report we show that a moderate neuronal overexpression of ADAM10 in mice transgenic for human APP $_{[V 7171]}$ increased the secretion of the neurotrophic soluble $\alpha$-secretase-released N-terminal APP domain (APPs $\alpha$ ), reduced the formation of $A \beta$ peptides, and prevented their deposition in plaques. Functionally, impaired long-term potentiation and cognitive deficits were alleviated. Expression of mutant catalytically inactive ADAM10 led to an enhancement of the number and size of amyloid plaques in the brains of double-transgenic mice. The results provide the first in vivo evidence for a proteinase of the ADAM family as an $\alpha$-secretase of APP, reveal activation of ADAM10 as a promising therapeutic target, and support the hypothesis that a decrease in $\alpha$-secretase activity contributes to the development of AD.
\end{abstract}

\section{Introduction}

Alzheimer disease $(\mathrm{AD})$ is a progressive neurodegenerative disorder characterized by the formation of amyloid $\beta$-peptides $(A \beta$ peptides) and their deposition in the brain as senile plaques (1). The $A \beta$ peptides $A \beta 40$ and $A \beta 42$, and especially their oligomeric aggregates, are believed to play a central role in $\mathrm{AD}$ by causing neurotoxicity $(2,3)$, development of neurofibrillary tangles (4), impairment of long-term potentiation (LTP) (5), and age-related cognitive deficits (6).

Strategies to treat $\mathrm{AD}$ are aimed at preventing the formation of $A \beta$ peptides. Therefore, $\beta$ - and $\gamma$-secretases that generate $A \beta$ peptides by sequential cleavage of the amyloid precursor protein (APP) are obvious and main targets for the development of specific inhibitors (7). Alternatively, increasing $\alpha$-secretase activity in the brain provides an attractive strategy, since proteolysis of APP within the $A \beta$ sequence precludes the formation of $A \beta$ peptides $(8-10)$. In addition, $\alpha$-secretase cleavage releases the $\mathrm{N}$-terminal extracellular domain known as soluble $\alpha$-secretase-released $\mathrm{N}$-terminal APP domain (APPs $\alpha$ ), which has neurotrophic and neuroprotective properties $(11,12)$. It is interesting to note that

Nonstandard abbreviations used: a disintegrin and metalloproteinase (ADAM); Alzheimer disease (AD); amyloid $\beta$-peptide (A $\beta$ peptide); amyloid precursor protein (APP); C-terminal fragment (CTF); hemagglutinin (HA); long-term potentiation (LTP); soluble $\alpha$-secretase-released $\mathrm{N}$-terminal APP domain (APPs $\alpha$ ); soluble $\beta$-secretase-released $\mathrm{N}$-terminal APP domain (APPS $\beta$ ); trifluoroacetic acid (TFA). Conflict of interest: The authors have declared that no conflict of interest exists. a reduction of APPs $\alpha$ is evident in the cerebrospinal fluid of AD patients $(13,14)$. The $\mathrm{p} 3$ peptide (A $\beta 17-42)$, derived by $\alpha$ - and $\gamma$-secretase cleavage of APP, is not generally found in amyloid cores of classical plaques or in amyloid deposits in the cerebral vasculature $(15,16)$. It accumulates predominantly in diffuse amyloid deposits in selected areas of the $\mathrm{AD}$ brain (17). In vitro, $A \beta 17-42$ has been reported to induce neuronal apoptosis, although with a lower potency than A $\beta 1-42(18,19)$. At present, it is not known whether an increase of $\alpha$-secretase activity in vivo has overall beneficial effects with regard to $\mathrm{AD}$ pathology.

Three members of the ADAM family (a disintegrin and metalloproteinase), ADAM9, ADAM10, and ADAM17, can act as $\alpha$-secretases in various cell lines (20-22). ADAM10 in particular has many properties of a physiologically relevant $\alpha$-secretase: it cleaves APP-derived peptides at the main $\alpha$-secretase cleavage site between position 16 and 17 of the $A \beta$ region, has $\alpha$-secretase activity in cultured cells, and is expressed in mouse and human brain $(21,23,24)$. ADAM10-deficient mice have been generated (25), but their early lethality prevented a reliable analysis of ADAM10 function in vivo, especially in neuronal cells. In particular, evidence is lacking that an increase in activity of putative $\alpha$-secretases prevents plaque formation and cognitive deficits.

The present study mainly addresses two questions. Does overexpression of ADAM10 in vivo increase the nonamyloidogenic processing of APP and increase APPs $\alpha$ while decreasing A $\beta$ levels and amyloid plaque formation? Will this overexpression also improve the synaptic plasticity and cognitive deficits in a mouse model for the amyloid pathology in $\mathrm{AD}$ ? 


\section{Methods}

Antibodies. Antibody 6E10 (Signet Laboratories Inc., Dedham, Massachusetts, USA) is directed against amino acids 1-16 of human A $\beta$ and recognizes APPs $\alpha$. 192wt (kindly provided by S. Sinha, Elan Pharmaceuticals, South San Francisco, California, USA) is directed against residues 591-596 of APP695, and detects only the soluble $\beta$-secretase-released $\mathrm{N}$-terminal APP domain (APPs $\beta$ ) (26). Y-11 (Santa Cruz Biotechnology Inc., Santa Cruz, California, USA) is directed against the hemagglutinin (HA) epitope. Anti-ADAM10 antibody (Chemicon International Inc., Temecula, California, USA) is directed against the C-terminus of ADAM10. 6F/3D (DAKO A/S, Glostrup, Denmark) is directed against residues $8-17$ of $A \beta 1-42$ and detects $A \beta 40$ and $A \beta 42.4 G 8$ (Signet Laboratories Inc.) is directed against residues 17-24 of $A \beta 1-42$, detecting whole $A \beta$ peptides, $\mathrm{p} 3$, and other $A \beta$ peptides truncated at the N-terminus. FCA3340 (EMD Biosciences Inc., San Diego, California, USA) is directed against amino acids 33-40 of $A \beta 1-40$ and detects the C-terminus of $A \beta X-40$ peptides (27). FCA3542 (EMD Biosciences Inc.) is directed against amino acids $35-42$ of $A \beta 1-42$, detecting the C-terminus of $A \beta X-42$ peptides (27). 6687 (kindly provided by C. Haass, Ludwig-Maximilians University Munich, Munich, Germany) is an antibody against the C-terminus of human APP.

Generation of transgenic mice. All animal husbandry procedures and experiments were performed in accord with the guidelines of the German Council on Animal Care. The cDNA's coding for C-terminally HA-tagged bovine ADAM10 and the dominant-negative form, ADAM10-E384A-HA (ADAM10-dn), were cloned under control of a neuron-specific mouse thy 1 promoter (28) kindly provided by Herman van der Putten (Novartis AG, Basel, Switzerland). The minigenes were microinjected into pronuclear embryos from superovulated $F V B / N$ females as described (29). Founders were identified by Southern blotting and by PCR and were mated to $F V B / N$ mice to establish heterozygous transgenic lines. Heterozygous offspring of $A D A M 10$ mice were intercrossed to generate homozygous $A D A M 10$ mice. For the generation of doubletransgenic mice, single-transgenic $A P P_{[V 717 I]}$ mice (strain $F V B / N$ ) (30) were intercrossed with $A D A M 10$ or $A D A M 10-d n$ animals.

Quantitative real-time RT-PCR. Total RNA from mouse brain was isolated using TriFast (Peqlab Biotechnologie GmbH, Erlangen, Germany). PCR primers were designed using Primer Express version 1.5 software (Applied Biosystems, Foster City, California, USA). Real-time PCR reactions were performed using $500 \mathrm{ng}$ RNA, 5 pmol of each primer, and the one-step QuantiTect SYBR Green RT-PCR kit (Qiagen GmbH, Hilden, Germany). Analysis was performed with ABI Prism 7000 and ABI Prism 7000 SDS software (Applied Biosystems). Differences in mRNA levels were calculated from variations in cycle thresholds.

Sample preparation from brains of transgenic mice. Brain hemispheres of 18-week-old mice were homogenized in 6.5 volumes of ice-cold buffer (20 mM Tris-HCl, $\mathrm{pH} 8.5$ ) containing a mixture of proteinase inhibitors (Roche Diagnostics Corp., Mannheim, Germany). After centrifugation at $135,000 \mathrm{~g}$ for 1 hour at $4^{\circ} \mathrm{C}$, the supernatant was used for quantitation of the soluble $A \beta$ peptides, APPs $\alpha$ and APPs $\beta$. Full-length APP, the APP C-terminal fragments (CTFs), and ADAM10 were quantified within the membrane pellet fraction.

Quantitation of amyloid peptides by ELISA. For isolation of soluble $A \beta, 80 \%$ of the brain homogenate supernatant (see above) and C18 Sep-Pak cartridges (Waters Corp., Milford, Massachu- setts, USA) were used. The C18 columns were equilibrated first with $80 \%$ acetonitrile/ $0.1 \%$ trifluoroacetic acid (TFA) and then with $0.1 \%$ TFA. Subsequently, soluble fractions of the brain homogenates were applied. After washing with increasing concentrations of acetonitrile (5\%, 25\%, and 50\%) containing $0.1 \%$ TFA, A $\beta$ peptides were eluted with $50 \%$ acetonitrile containing $0.1 \%$ TFA. Eluates were lyophilized and then $A \beta$ peptides were dissolved for quantification by ELISA.

Human $A \beta 40$ peptides were quantified by sandwich ELISA as described (31). Briefly, A $\beta 40$ peptides were captured with a specific antibody directed against the C-terminus of the $\mathrm{A} \beta$ peptide, and detection was performed with an antibody directed against amino acids $1-16$ of $A \beta 1-40$. Human $A \beta 42$ peptides were quantified using a commercial ELISA assay (Innogenetics, Ghent, Belgium) whereby a specific antibody directed against the $\mathrm{C}$-terminus of $A \beta 42$ peptides is used for capturing and an antibody directed against amino acids $1-5$ of $A \beta 1-42$ is used for detection.

Analysis of secreted APP-processing products. APPs $\alpha$ and APPs $\beta$ were analyzed by Western blot using 7.5\% SDS-PAGE gels and $200 \mu \mathrm{g}$ protein per lane. APPs $\alpha$ and APPs $\beta$ were detected with antibodies 6E10 and 192wt, respectively. For detection and quantitation, ${ }^{35}$ S-labeled secondary antibodies (Amersham Biosciences, Freiburg, Germany) were used. Bound radioactivity was detected with the Bio-Imaging Analyzer BAS-1800 (FujiFilm Medical Systems USA Inc., Stamford, Connecticut, USA) and quantified with the software AIDA 2.0 (Raytest GmbH, Straubenhardt, Germany).

Quantitation of membrane-bound APP-processing products and ADAM10. Full-length APP, APP CTFs, and HA-tagged ADAM10 were analyzed in $25 \mu \mathrm{g}$ of mouse brain membrane protein per lane using $4-12 \%$ Bis-Tris NuPAGE gradient gels (Invitrogen Corp., Carlsbad, California, USA) and Western blotting. Full-length APP and APP CTFs were detected with antibody 6687. HA-tagged ADAM10 proteins were detected with the antibody Y-11. Detection and quantitation was performed as described above.

Quantitation of ADAM10 expression levels. ADAM10 proteins were enriched by chromatography on concanavalin A-agarose as described (32) using Triton X-100 for solubilization of mouse brains. Mature ADAM10 was quantified by Western blotting using $10 \%$ SDS PAGE gels and $200 \mu \mathrm{g}$ purified glycoproteins per lane. Quantitation was performed as described above using an anti-ADAM10 antibody.

Immunobistochemistry and quantitative plaque analysis. Brains were dissected and cut sagittally. One hemisphere was fixed with $4 \%$ paraformaldehyde in PBS for 24 hours and was then cut frontally. The frontal quarter was embedded in paraffin and sections for histological analysis were prepared according to standard protocols. HA-tagged ADAM10 was detected with antibody Y-11 and amyloid plaques were identified with the antibody $6 \mathrm{~F} / 3 \mathrm{D}$, detecting $\mathrm{A} \beta 40$ and $A \beta 42$, applying the alkaline phosphatase anti-alkaline phosphatase (APAAP) method with fuchsin as substrate following the manufacturer's protocol (DAKO A/S). Immunostainings with antibodies FCA3340, FCA3542, and 4G8 were also performed.

The occipital paraformaldehyde-fixed quarter was used for quantitation of the amyloid plaque load in the subiculum using thioflavine $\mathrm{S}$ staining of vibratome-cut sections (40 $\mu \mathrm{m}$ thick) as described (31). Well-defined coronal sections at bregma $-3.52 \mathrm{~mm}$ (33) were stained with thioflavine $\mathrm{S}$ and selected for quantitation of the amyloid load in the subiculum. Fluorescence images were acquired on an inverted microscope (Leica DMR; Leica Microsystems, Bensheim, Germany) equipped with a 3 CCD digi- 

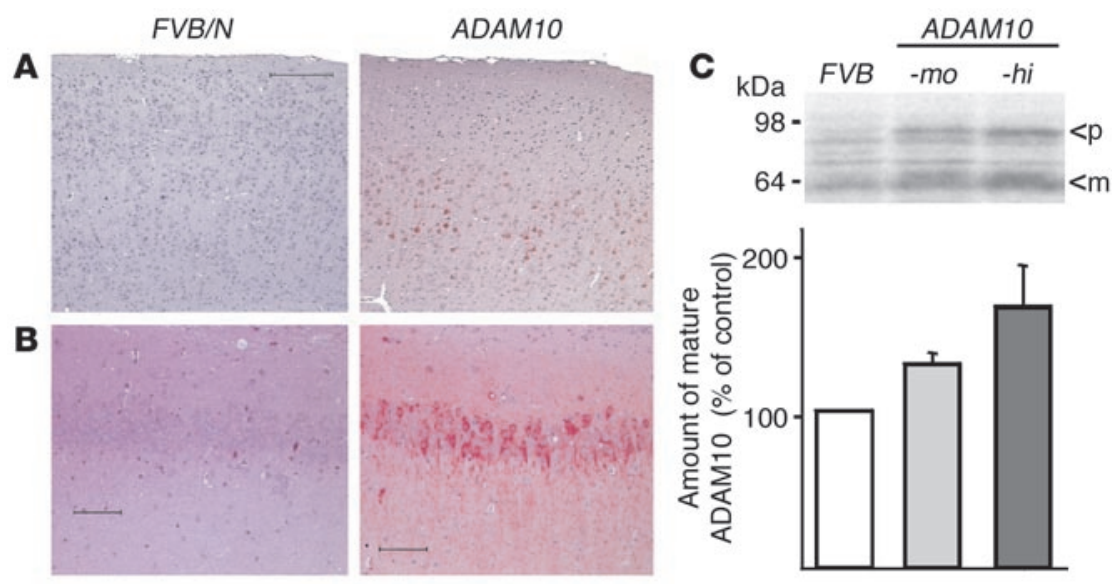

Figure 1

Characterization of $A D A M 10$ transgenic mice. Immunohistochemical detection of HA-tagged ADAM10 using the antibody Y-11. Cortex (A) and hippocampal (B) sections of ADAM10-mo transgenic animals and a nontransgenic control $(F V B / N)$ are shown. Scale bars: $200 \mu \mathrm{m}$ in A and $100 \mu \mathrm{m}$ in B. (C) Quantitation of catalytically active ADAM10 protein levels. Enriched ADAM10 proteins from adult mouse brains were subjected to Western blot analysis and detected with an anti-ADAM10 antibody. The upper panel shows mature ADAM10 $(<\mathrm{m})$ and the proform of ADAM10 $(<\mathrm{p})$. The mature 62-kDa ADAM10 form was quantified using a ${ }^{35}$ S-labeled secondary antibody (lower panel).

tal camera (Sony DXC-9100P; Sony Corp., Cologne, Germany), and analyzed with dedicated software (Leica QWin system). Light intensity and condenser settings for the microscope were carefully kept constant throughout the entire image acquisition process. Density slice threshold was applied uniformly throughout analysis. The total surface area of amyloid deposits was measured and expressed as a percentage of the total surface of the subiculum.

Electrophysiology. Hippocampal slices (400 $\mu \mathrm{m}$ thick) were prepared from ether-anesthetized 6-to 7-month-old mice using a vibratome. After a recovery period of at least 1.5 hours, slices were incubated in a submersion chamber perfused at a rate of 3 $\mathrm{ml} / \mathrm{min}$ with warm $\left(30^{\circ} \mathrm{C}\right)$, oxygenated $\left(95 \% \mathrm{O}_{2} / 5 \% \mathrm{CO}_{2}\right)$ artificial cerebrospinal fluid. A bipolar tungsten microelectrode was used to stimulate the Schaffer collaterals pathway. The evoked field excitatory postsynaptic potentials were recorded in the stratum radiatum of the CA1 region with a low-resistance ( $2 \mathrm{M} \Omega$ ) glass microelectrode filled with $2 \mathrm{M} \mathrm{NaCl}$. The test stimulation (monophasic, $0.1 \mathrm{~ms}$ duration) was delivered once per minute. For inducing LTP, two trains of stimulation at $100 \mathrm{~Hz}$ for $1 \mathrm{sec}-$ ond, 20 seconds apart, were delivered at the same intensity as the test stimulation (biphasic pulses, $0.1 \mathrm{~ms}$ per phase).

Morris water maze. Adult male mice ( $n=9-10$ for each group; strain background, $\mathrm{FVB} / \mathrm{N}-\mathrm{C} 57 \mathrm{BL} / 6$ ) aged 6-10 months were trained on a Morris water-maze task using a submerged platform following standard procedures (34). Prominent objects around the maze provided abundant extra-maze cues. Mice performed four trials per day (one session) for 4 days with a maximum length of 90 seconds and an intertrial interval of 90 seconds. The platform was in the same quadrant for all trials and the animals were placed randomly. A computerized video system (EthoVision; Noldus Information Technology, Utrecht, The Netherlands) registered swim path and duration. We allowed the mice to stay on the platform for 30 seconds. On the fifth day we performed a probe trial (60 seconds) without the platform. The time to reach the location of the supposed platform was measured and presented as latency. In addition, the number of annulus crossings was calculated.

\section{Results}

Generation and characterization of ADAM10overexpressing mouse lines. As a first step to explore the activity of ADAM10 in vivo, we generated transgenic mice expressing - under the control of a neuron-specific postnatally active mouse thy 1 promoter (28) - either ADAM10 with a C-terminal HA tag or its catalytically inactive form. The inactive form of ADAM10 has the point mutation E384A in the zincbinding motif. In cultured cells, HA-tagged ADAM10 has been shown to retain $\alpha$-secretase activity, and the E384A mutant has been shown to inhibit generation of APPs $\alpha$ (21). Three transgenic mouse lines overexpressing ADAM10 were selected and characterized. Furthermore, one line expressing the E384A mutant of ADAM10 (ADAM10-dn) was included on the assumption that it might interfere with endogenous $\alpha$-secretase-mediated APP processing. By Western and Northern blot analysis, moderate expression levels of HAtagged ADAM10 were shown in two lines, and one line (ADAM10-hi) expressed higher levels of the protein (data not shown). One line expressing moderate levels of ADAM10 (ADAM10-mo) was selected for further study and was also bred to generate homozygous offspring (ADAM10-hz). All ADAM10 transgenic mice were inconspicuous in morphology, breeding, and in daily handling.

The expression of HA-tagged ADAM10 proteins in transgenic mouse brains was demonstrated by immunohistochemistry to be restricted to neurons. As expected for the thy 1 promoter used in this study, we detected the highest levels in several layers of the neocortex and in large pyramidal neurons in the hippocampus (Figure 1, A and B).

To determine the expression of catalytically active transgenic ADAM10 relative to endogenous ADAM10, we enriched glycosylated proteins of brains from adult animals by concanavalin A-agarose chromatography and subjected them to Western blot analysis using an antibody that recognizes endogenous murine and transgenic bovine ADAM10. Both mature 62-kDa ADAM10 and the $90-\mathrm{kDa}$ proform were detected (Figure 1C). The purified $62-\mathrm{kDa}$ protein has been shown to act as an $\alpha$-secretase in vitro and to cleave $A \beta$-derived peptides between Leu ${ }^{16}$ and Lys ${ }^{17}(21)$. By quan titative analysis we found a $30 \%$ increase of mature ADAM 10 in line ADAM10-mo and a 70\% increase in line ADAM10- $h i$ compared with nontransgenic $F V B / N$ mice (Figure $1 C$ ). In the line expressing the E384A mutant of ADAM10, a sevenfold increase of the catalytically inactive ADAM10 protein compared with endogenous ADAM10 was determined by the same procedure.

There is evidence that ADAM10 plays a role in the Notch signaling pathway during neural development in embryonic mice (25). In mouse brains, very low levels of the Notch intracellular domain are beyond the detection limit, thus precluding its direct analysis. We therefore examined whether Notch signaling was affected by analyzing the expression level of Hes5, a gene affected downstream of Notch (35). Quantitative real-time RT-PCR experiments on total 
A

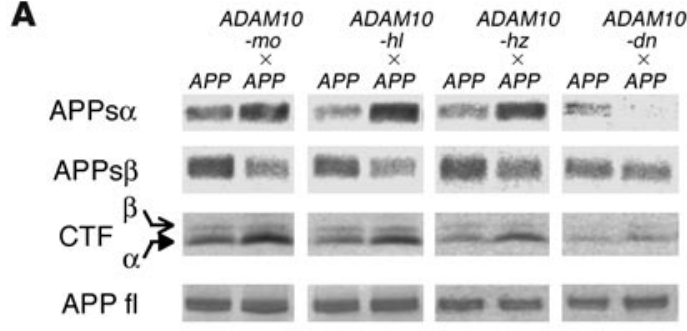

B
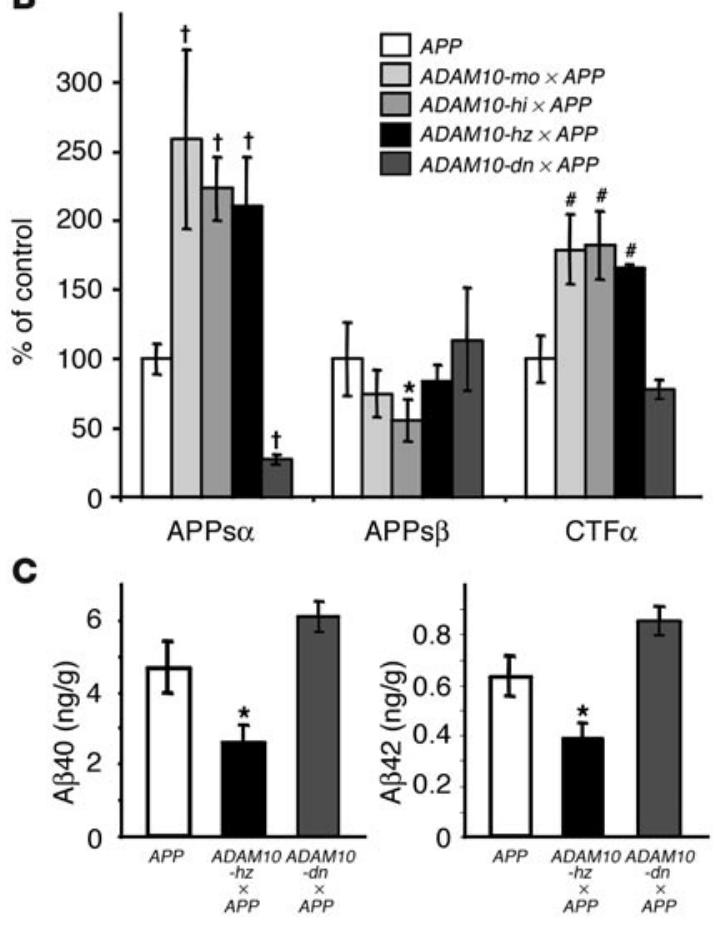

RNA from brains of mice ( $n=5$ of each line) at the age of 10 weeks revealed no significant differences $(P=0.1)$ between Hes 5 transcript levels of nontransgenic mice and ADAM10-overexpressing mice.

Effect of ADAM10 on APP processing. To investigate the influence of ADAM10 on APP processing in detail, including its effect on amyloid plaque formation and LTP, we generated double-transgenic mice by crossing the ADAM10-overexpressing lines (ADAM10-mo, ADAM10-hi, and ADAM10-hz) as well as the line expressing mutant ADAM10-dn with mice transgenic for human $\mathrm{APP}_{[\mathrm{V} 717]]}$. The $A P P_{[V 717]}$ transgenic line expresses mutant human APP under control of the same thy1 promoter as used for transgenic ADAM10 expression. In this line increased levels of $A \beta$ peptides are detectable around 3 months after birth, followed by defects in LTP and cognition. Furthermore, cortical and hippocampal amyloid plaques and vascular pathology are evident at the age of 12 months $(30,31,36)$.

First we verified that in all double-transgenic mouse lines, the two transgenes do not regulate each other's expression level. To do this, ADAM10 proteins of double-transgenic mice were enriched by lectin chromatography and quantified by Western blotting. This analysis confirmed that in double-transgenic $A D A M 10 \times A P P_{[V 717]}$ mice, catalytically active ADAM 10 proteins were expressed at the same level as in the corresponding parental lines. In $A D A M 10-h z \times A P P_{[V 717]}$ mice, ADAM10 expression was 2.2-fold higher than the endogenous level (not shown). Quantitation of human $\mathrm{APP}_{[\mathrm{V717]}}$ expression in dou-

\section{Figure 2}

Detection and quantitation of human APP processing products in double-transgenic ADAM10-mo $\times A P_{[V 717 l]}, A D A M 10-h i \times A P P_{[V 717]]}$, $A D A M 10-h z \times A P P_{[V 717]}$, and $A D A M 10-d n \times A P P_{[V 717]]}$ mice as well as in monotransgenic $A P P_{[V 717]]}(A P P)$ animals. All analyzed mice were 18 weeks old. (A) APP processing products detected by Western blot analysis as described in Methods. First row, secreted APPs $\alpha$; second row, secreted APPs $\beta$; third row, membrane-bound APP C-terminal fragments (CTF $\alpha, C T F \beta)$; last row, membrane-bound full-length APP (APP fl). (B) Quantitative analysis of the APP-processing products APPs $\alpha, A P P s \beta$, and APP CTF $\alpha$ from different mouse lines. In all experiments, quantified APP processing products were normalized to APP fl expression. Values are expressed as percentages of values from APP control mice (set to 100\%) and are the mean \pm SD $(n=7-8$ animals of each line). Significance was determined by the unpaired Student's $t$ test. ${ }^{*} P<0.05$; ${ }^{\sharp} P<0.01 ;{ }^{\dagger} P<0.001$. (C) Quantitation of soluble human $A \beta 40$ and $A \beta 42$ peptides isolated from mouse brains by sandwich ELISA. $A P P_{[V 717]]}$ mice $(n=6)$ were used as control to $A D A M 10-h z \times \operatorname{APP}_{[V 717]]}(n=7)$ and $A D A M 10-d n \times \operatorname{APP}_{\text {[V717] }}(n=6)$ animals. Values are the mean \pm SEM of the amount of each $A \beta$ peptide per gram of mouse brain. Significance was determined by the unpaired Student's $t$ test. ${ }^{*} P<0.05$.

ble-transgenic $A D A M 10 \times A P P_{[V 717]]}$ and monotransgenic $A P P_{[V 117]}$ mice revealed no significant variation of the level of full-length APP (Figure 2A, last row). This result was further confirmed by quantitative real-time RT-PCR studies (not shown) indicating that transgenic expression of ADAM10 and APP, both under control of the same promoter, do not influence each other.

As APP is a substrate for both the putative $\alpha$-secretase ADAM10 and for the $\beta$-secretase BACE1, the expression levels of endogenous murine ADAM10 and BACE1 might be altered in ADAM10 $\times A P P$ double-transgenic mice. By quantitative real-time RT-PCR experiments, we could not detect differences in brain mRNA levels between double-transgenic ADAM10-mo $\times A P P_{[V 717 I]}, A D A M 10-d n \times$ $A P P_{[V 717]}$, and $A P P_{[V 117]}$ monotransgenic mice (not shown).

Changes in APP processing can be detected in $A P P_{[V 717]}$ transgenic mice by the early age of 3 months (30); therefore, we analyzed brain samples of 18-week-old double-transgenic ADAM10 $\times A P P_{[V 717 I]}$ animals and monotransgenic $A P P_{[V 717]}$ control mice. As expected with enhanced $\alpha$-secretase activity, overexpression of ADAM10 strongly augmented both the levels of secreted APPs $\alpha$ and the membrane-bound $\alpha$-secretase-cleaved APP CTF (CTF $\alpha)$ (Figure 2A). Quantitation revealed that the level of neuroprotective APPs $\alpha$ increased more than twofold in all three double-transgenic lines overexpressing ADAM10. The level of the corresponding CTF $\alpha$ was increased somewhat less, approximately 1.7 -fold, which is presumably due to further cleavage by $\gamma$-secretase (Figure 2B). We also observed a small reduction in the amount of $\beta$-secretase-cleaved secreted APPs $\beta$ (Figure 2, A and B), indicating that there is competition between the two major proteolytic pathways acting on APP in vivo.

Compared with CTF $\alpha$, the amount of CTF $\beta$ was already low in $A P P_{[V 717]}$ transgenic animals, thus preventing reliable quantitation of any changes in double-transgenic animals (Figure 2A).

Analysis of brain homogenates from mice overexpressing dominant-negative ADAM10 revealed that the $\alpha$-secretase pathway was strongly inhibited, as concluded from the $75 \%$ reduction in APPsa levels (Figure 2, A and B). Surprisingly, the quantity of CTF $\alpha$ was only slightly decreased, by about $20 \%$, indicating that the CTF $\alpha$ in $A D A M 10-d n \times A P P_{[V 717 I]}$ transgenic mice is more resistant to further proteolytic degradation than in 

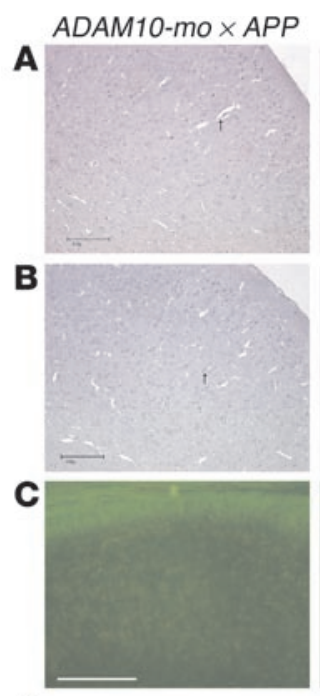

D

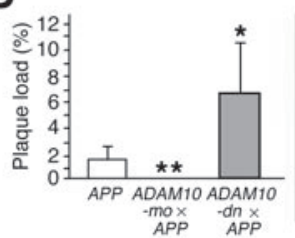

$A D A M 10-d n \times A P P$
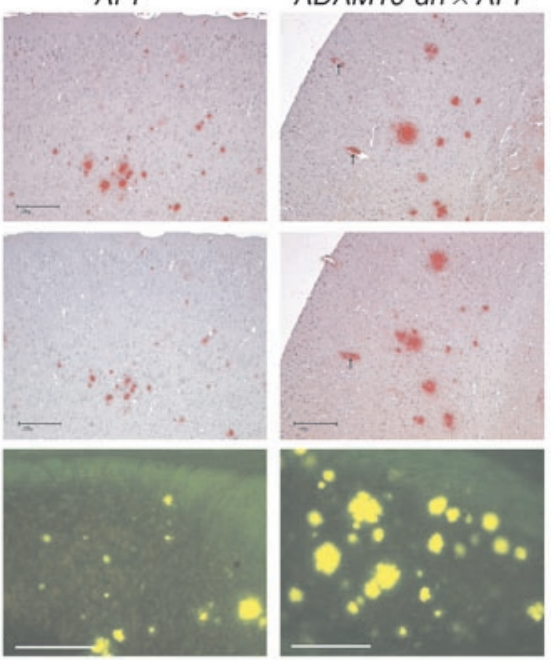

E

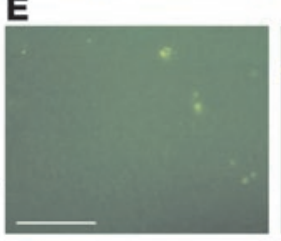

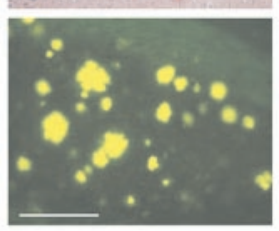

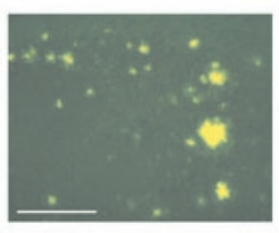

$A P P_{[V 717]]}$ mice. Quantitation of APPs $\beta$ revealed a small increase in $A D A M 10-d n \times A P P_{[V 717 I]}$ mice compared with $A P P_{[V 717 I]}$ control animals (Figure $2 \mathrm{~B}$ ).

The levels of the APP-derived soluble peptides $A \beta 40$ and $A \beta 42$ in brains of double-transgenic mice and $A P P_{\text {[V717I] }}$ control animals at the age of 18 weeks were quantified by specific sandwich ELISAs. In all lines overexpressing ADAM10, the amounts of $A \beta 40$ and $A \beta 42$ were decreased relative to those in mice of the parental $A P P_{[V 717 I]}$ line. We found that in line $A D A M 10-b z \times$ $A P P_{[V 717 I]}$, the amounts of both peptides were reduced by about $44 \%$ and $40 \%$, respectively (Figure $2 \mathrm{C}$ ). In line ADAM10-mo $\times$ $A P P_{[V 717 I}, \mathrm{A} \beta 40$ and $\mathrm{A} \beta 42$ were reduced by $49 \%$ and $2 \%$, and in line $A D A M 10-h i \times A P P_{[V 717 I]}$, by $39 \%$ and $29 \%$, respectively. In brains of mice that overexpress mutant ADAM10-dn and $\mathrm{APP}_{[\mathrm{V717I}]}$, both peptides were increased by about 30\% (Figure 2C).

In the parental $A P P_{[V 717]}$ transgenic line, amyloid plaque formation is caused by increased levels of $A \beta$ peptides. Plaque load is evident at the age of 12 months and reaches its maximum within 15 months of age (30). Therefore, we analyzed plaque formation in all double-transgenic $A D A M 10 \times A P P_{[V 717 I]}, A D A M 10-d n \times A P P_{[V 717 I]}$, and $A P P_{[V 717 I]}$ control animals when they were $15-19$ months old.

\section{Figure 4}

Analysis of amyloid deposits in brains from 17- to 19-month-old double-transgenic $A D A M 10-m o \times A P P_{[V 717 l]}(\mathbf{A}$ and $\mathbf{B})$ and $A D A M 10-$ $d n \times A P P_{[V 717 !]}$ mice $(\mathbf{C}$ and $\mathbf{D})$. Isolated tiny $(\mathbf{A})$ and diffuse (B) amyloid deposits in the brain of an ADAM10-mo $\times A P P_{[V 717]}$ mouse. Immunohistochemistry was performed with antibodies 6F/3D (A) and 4G8 (B), detecting either only $A \beta$ peptides or $A \beta$ peptides in addition to $\mathrm{N}$-terminally truncated $A \beta$ peptides ( $p 3$ fragments). Scale bars: 100 $\mu \mathrm{m}$. (C and $\mathbf{D})$ Analysis of amyloid plaque composition in the brain of an $A D A M 10-d n \times A P P_{[V 717]}$ mouse. Immunohistochemistry was performed with antibodies FCA3542 (C) and FCA3340 (D), detecting peptides containing $A \beta X-42$ and $A \beta X-40$, respectively. Antibody FCA3340 detects fewer plaques than FCA3542 does. Scale bars: $200 \mu \mathrm{m}$.

\section{Figure 3}

Detection and quantitation of amyloid plaques in brains from 17- to 19-month-old (A-D) $A P P_{[V 717 \mid]}$ transgenic mice, doubletransgenic $A D A M 10-m o \times A P P_{[V 717]]}$ mice, and ADAM10-dn $\times$ $A P P_{[V 717]]}$ mice. Immunohistochemical detection of amyloid plaques in the neocortex in paraffin-embedded sections with either antibody 6F/3D (A) or antibody 4G8 (B). Note that in ADAM10-mo $\times$ $A P P_{[V 717]}$ mice, no additional plaques were detected by antibody 4G8. Arrows point to blood vessels. Scale bars: $200 \mu \mathrm{m}$. (C and E) Thioflavine $S$-stained $\beta$ structures in the subiculum of either 17- to 19-month-old (C) or 12-month-old mice (E); scale bars: 200 $\mu \mathrm{m}$. (D) Quantitation of amyloid load in subiculum in thioflavine S-stained sections obtained from 17- to 19-month-old animals. Surface thioflavine $S$ staining is expressed as a percentage of the total subiculum surface. Statistical analysis was performed for each genotype with the following number of animals: $A P P_{[V 717]}, n=5$; $A D A M 10-m o \times A P P_{[V 717 l]}, n=13 ; A D A M 10-d n \times A P P_{[V 717 l]}, n=6$. ${ }^{\star} P<0.05 ;{ }^{* *} P<0.01$.

For immunohistology on cortical brain areas, we used several antibodies to characterize the composition of amyloid deposits. Antibody $6 \mathrm{~F} / 3 \mathrm{D}$ detects amyloid peptides $\mathrm{A} \beta 40$ and $\mathrm{A} \beta 42$, but not the $\mathrm{p} 3$ fragments $A \beta 17-40$ and $A \beta 17-42$. To investigate whether overexpression of ADAM10 may lead to the deposition of $\mathrm{p} 3$ fragments, additional immunostainings were performed using the antibody 4G8 and antibodies directed against the C-terminus of either $\mathrm{A} \beta X-40$ or $\mathrm{A} \beta X-42$ peptides (FCA3340 and FCA3542, respectively). These three antibodies recognize $\mathrm{p} 3$ fragments in addition to $\mathrm{A} \beta 40$ and $\mathrm{A} \beta 42$.

We found that in $A P P_{[V 717]]}$ control animals, plaque load did not increase significantly in the time interval between 15 and 19 months. The majority of mice coexpressing ADAM10 together with $\mathrm{APP}_{[\mathrm{V7171}]}$ developed no plaques (Figure $3, \mathrm{~A}-\mathrm{C}$ ), while in some (6 of 20) we found only isolated tiny or diffuse amyloid deposits

A

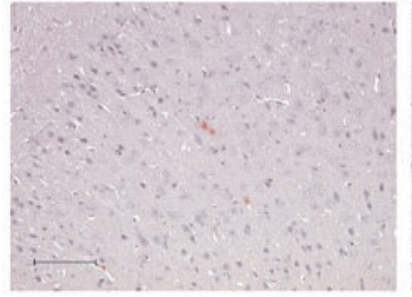

C

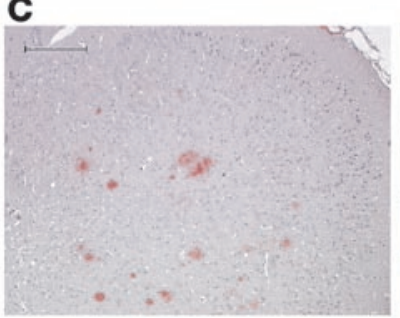

B

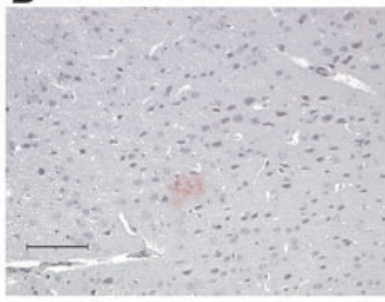

D

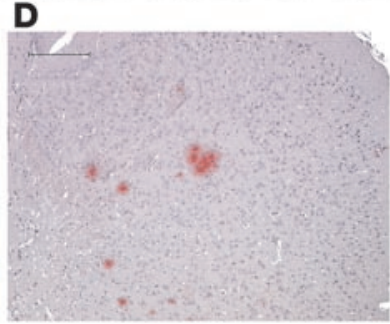


A

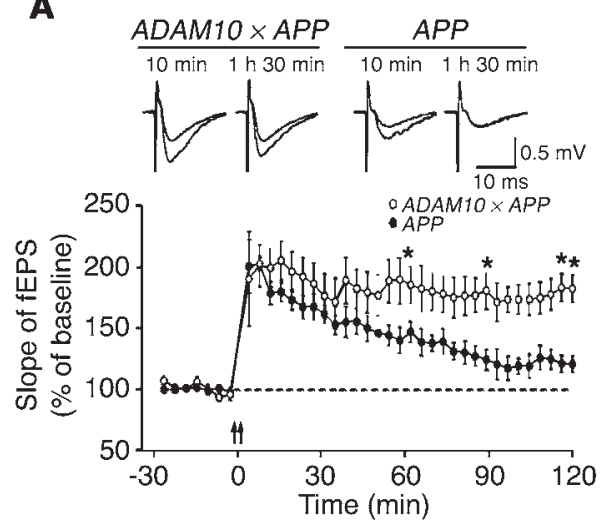

\section{Figure 5}

LTP studies of hippocampal slices of transgenic mice. (A) LTP in hippocampal slices. The slope of the field excitatory postsynaptic potential (fEPSP) is plotted as a function of time before and after high-frequency stimulation in slices from $A P P_{[V 717]}$ mice (filled circles, $n=6$ ) and from ADAM10-hz $\times A P P_{[V 717]]}$ mice (open circles, $n=5$ ). The two arrows indicate the time at which the two tetani were applied. Samples of traces obtained from $A D A M 10-h z \times A P P_{[V 717]]}$ and $A P P_{[V 717]]}$ mice are shown at the top. Recordings of fEPSPs obtained either 10 minutes or 1 hour and 30 minutes after tetanic stimulation are superimposed on the same control trace obtained before stimulation. (B) Input-output curve of fEPSP amplitude $(\mathrm{mV})$ versus stimulus $(\mathrm{V})$ at the Schaffer collaterals did not differ significantly between genotypes. (C) Paired-pulse facilitation did not differ between $\operatorname{APP}_{[V 717]]}(n=6)$ and $A D A M 10-h z \times \operatorname{APP}_{[V 717]}(n=7)$ mice at the tested interval of $50 \mathrm{~ms}$. Traces showing typical paired-pulse facilitation in an $A P P_{[V 717 l]}$ and in an $A D A M 10-h z \times A P P_{[V 717 l]}$ mouse are shown on the right. Significance was determined by unpaired Student's $t$ test. ${ }^{\star} P<0.01$.

(Figure 4, A and B), demonstrating that increased ADAM10 activity almost completely prevents amyloid plaque formation. This phenomenon was observed with only a moderate overexpression of ADAM10 (Figure 3, A-C) and was independent of the sex and age of the animals in the investigated time interval.

Antibodies recognizing $N$-terminally truncated $A \beta$ peptides (e.g., 4G8; Figure 3B) did not detect additional amyloid deposits compared with an antibody directed against an epitope located at the $\mathrm{N}$-terminal site of the $\alpha$-secretase cleavage position (Figure $3 \mathrm{~A})$, demonstrating that ADAM10 overexpression does not lead to deposition of $\mathrm{p} 3$ fragments.

In contrast, double-transgenic mice expressing mutant ADAM10dn clearly developed larger amyloid plaques (diameter up to 130 $\mu \mathrm{m})$ in amounts severalfold higher than were found in $A P P_{\text {[V717I] }}$ transgenic mice (Figure 3, A and B). Furthermore, both $\mathrm{A} \beta X-40$ and $A \beta X-42$ peptide isoforms were found in deposited plaques with specific antibodies. The antibody against the C-terminus of $\mathrm{A} \beta X-42$ detected more plaques than did the anti-A $\beta X-40$ antibody (Figure 4, $\mathrm{C}$ and $\mathrm{D})$. In this respect, line $A D A M 10-d n \times A P P_{[V 7171]}$ behaves like the parental $A P P_{[V 717 I]}$ line, in which plaques stained more intensely with the anti-A $\beta X-42$ antibody FCA3542 than with the anti-A $\beta X-40$ antibody FCA3340 (30). The cerebral amyloid angiopathy seen in parental transgenic $A P P_{[V 717 I]}$ mice (36) was also observed in ADAM10-dn $\times A P P_{[V 717]]}$ double-transgenic mice but was absent in ADAM10-mo $\times$ $A P P_{[V 717 I]}$ double-transgenic animals (Figure 3, A and B).
For quantitation of the amyloid plaque load we focused on the hippocampal subiculum area, because in the parental $A P P_{[V 717 I]}$ line, massive amyloid plaque formation can be detected in that area by immunohistochemistry and by thioflavine $\mathrm{S}$ staining (31). In all double-transgenic $A D A M 10 \times A P P_{\text {[V717I] }}$ lines, thioflavine $S$-stained $\beta$ structures were of negligible quantity, but in mice expressing mutant ADAM10-dn together with $\mathrm{APP}_{[\mathrm{V7171}}$, the amyloid plaque load was fourfold higher than that in the parental $\mathrm{APP}_{\text {[V717I] }}$ line (Figure 3, C and D). In addition to enhanced plaque formation, amyloid deposits occurred earlier in ADAM10-dn-expressing animals than in $A P P_{\text {[V717I] }}$ mice, as we observed a high plaque load at 12 months of age in $A D A M 10-d n \times A P P_{[V 717]}$ mice (Figure 3E).

Taken together, even relatively minor changes in the generation of $\mathrm{A} \beta$ peptides due to ADAM10 or mutant ADAM10 overexpression in brains of young APP-transgenic animals led to substantial differences in amyloid deposition over longer time periods: overexpression of ADAM10 clearly delayed senile plaque formation, and, in contrast, inhibition of the $\alpha$-secretase pathway resulted in a faster and more pronounced plaque formation in the mouse model used.

Effects of ADAM10 on LTP and memory. In human AD patients and in animal models, the hippocampal formation is one of the most affected CNS areas. Therefore, we addressed the question of whether typical hippocampal defects previously demonstrated in the parental $A P P_{[V 717 I]}$ transgenic line $(30,31)$ were alleviated in $A D A M 10 \times A P P_{[V 717 I]}$ double-transgenic animals expressing both transgenes in hippocampal neurons.

LTP, an electrophysiological correlate of synaptic plasticity, was measured at the level of synapses between the Schaffer collaterals and CA1 pyramidal cells in brain slices of transgenic mice. Tetanic stimulation triggered a significantly impaired LTP in $A P P_{[V 717 I]}$ transgenic mice, confirming previous findings $(30,31)$. This effect was corrected by overexpression of ADAM10 (Figure 5A). Immediately after its induction, the potentiation of the field excitatory postsynaptic potential slope reached similar levels in both groups $(200 \% \pm 22 \%$ SEM versus $190 \% \pm 38 \%$, expressed as percent of baseline). However, 2 hours later, this potentiation, which still reached $182 \% \pm 11 \%$ in slices from $A D A M 10-h z \times A P P_{[V 717 I]}$ mice, was only $120 \% \pm 7 \%$ in $A P P_{[V 717]]}$ transgenic animals $(P<0.01) . A D A M 10-b z \times A P P_{\text {[V717I] }}$ mice were back to normal conditions, as the field excitatory postsynaptic potential slope in nontransgenic control animals (not shown in Figure 5) was $196 \% \pm 10 \%(P=0.38)$. The relationship between stimulation intensity and the amplitude of the response by which basic excitability and synaptic transmission can be tested was similar in both groups (Figure 5B). Paired-pulse facilitation related to a very short-term presynaptic mechanism was also the same in both groups (Figure 5C).

Finally, cognitive characteristics of the mice were analyzed more globally by the Morris water-maze test, the most widely accepted behavioral test of hippocampus-dependent spatial learning and memory (37). Because $F V B / N$ mice are not optimal for this task due to vision defects (38), F1 hybrid mice from transgenic mice and $C 57 B L / 6$ animals were generated and investigated. Heterozygous $A P P_{[V 717 I]}$ transgenic mice in the same strain background devel- 
A

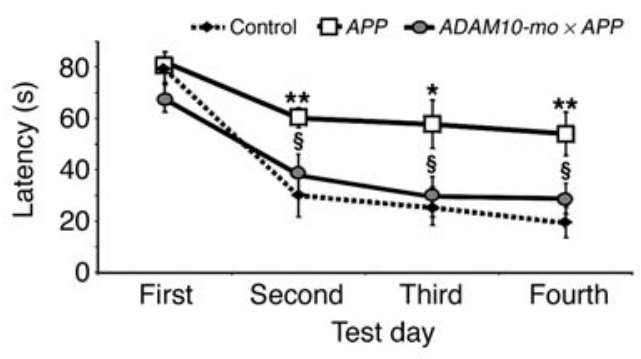

B

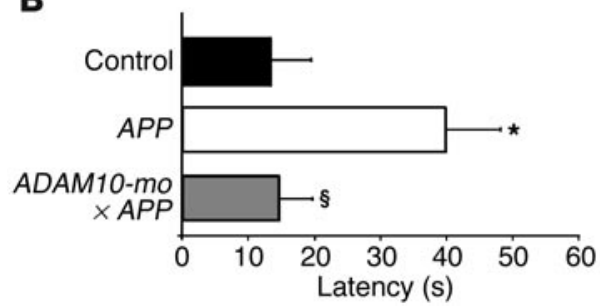

\section{Figure 6}

Morris water-maze. (A) Acquisition of place learning in the Morris water-maze hidden-platform task. Learning deficit in $A P P_{\text {[V717l] }}$ transgenic mice was ameliorated in double-transgenic ADAM10-mo $\times A P P_{[V 717]]}$ mice. Lines represent mean \pm SEM for 9-10 mice per group. ${ }^{\star} P<0.05,{ }^{\star \star} P<0.01, A P P_{[V 7171]}$ vs. control; $\$ P<0.05 A P P_{[V 7171]}$ vs. $A D A M 10-m o \times A P P_{[V 717]]}$ by Student's $t$ test. (B) Memory test in Morris water-maze probe trial without platform. The deficit in $A P P_{\text {[V717]] }}$ transgenic mice was improved in double-transgenic ADAM10-mo $\times$ $A P P_{[V 717 !]}$ mice. Bars represent mean \pm SEM for 9-10 mice per group. ${ }^{\star} P<0.05, A P P_{[V 7171]}$ vs. control; $\$ P<0.05, \mathrm{APP}_{[\mathrm{V} 717]]} \mathrm{Vs}$. ADAM10-mo $\times A P P_{[V 717]]}$ by Student's $t$ test.

op an amyloid pathology by the age of 12 months (van Leuven, unpublished data), but defects in hippocampus-dependent learning are already evident at the age of 6-10 months (30). In the Morris water maze, observed deficits in the acquisition phase of place learning and in the probe trial were not due to visual problems, as demonstrated in the cued navigation (visible platform) test in which $\mathrm{APP}_{[\mathrm{V717I}]}$ mice and nontransgenic hybrid mice displayed identical escape latencies (30).

We compared spatial learning and memory between male $A P P_{[V 717]}$ transgenic, double-transgenic ADAM10-mo $\times A P P_{[V 717 I]}$, and nontransgenic $F V B / N-C 57 B L / 6$ control mice. The time for reaching the location of the submerged platform was measured and presented as latency. As has been reported before $(30,31)$, $A P P_{[V 717]}$ transgenic mice were impaired in learning compared with nontransgenic animals (Figure 6A). Two-way ANOVA revealed a significant difference in latency resulting from genotype [factor one; $\mathrm{F}(2,25)=6.35, P<0.01$ ], and for the test day [factor two; $\mathrm{F}(3,75)=46.22, P<0.001]$ as well as for the genotype-session interaction $[\mathrm{F}(6,75)=2.27, P<0.05]$. The $A P P_{[V 717 I]}$ transgenic mice were also deficient in retaining the learned position, as revealed in a test trial without platform (Figure 6B): a significant difference in probe trial escape latency $[\mathrm{F}(2,25)=4.87, P<0.05]$ and probe trial annulus crossings $[\mathrm{F}(2,25)=384, P<0.05]$ (not shown) was observed between $A P P_{[V 717 I]}$ transgenic mice and double-transgenics overexpressing ADAM10. Both learning and memory were nearly fully restored in $A P P_{[V 7171]}$ mice moderately overexpressing ADAM10 (Figure 6, A and B): these animals needed significantly less time to reach the platform location. The distinct genotypes were not significantly different with regard to swimming speed (nontransgenic mice: $20.7 \pm 0.6 \mathrm{~cm} / \mathrm{s} ; A P P_{[V 717 I]}$ mice: $17.9 \pm 1.1$ $\mathrm{cm} / \mathrm{s} ; A D A M 10-m o \times A P P_{[V 717 I]}$ mice: $17.5 \pm 1.4 \mathrm{~cm} / \mathrm{s}$ ), demonstrating that neither locomotion nor motivation were affected.

\section{Discussion}

Our finding that neuronal overexpression of ADAM10 augments the $\alpha$-secretase cleavage of APP provides the first evidence that a proteinase of the ADAM family acts as an effective $\alpha$-secretase in vivo. We particularly note that even a moderate increase in ADAM10 expression strongly stimulates the $\alpha$-secretase processing of APP, delays plaque formation, and alleviates cognitive defects in a transgenic $\mathrm{AD}$ mouse model. Our observation that higher expression of ADAM10 does not further increase the amount of $\alpha$-secretase cleavage products may be explained by a limited amount of the substrate APP at the cell surface, where cleavage by the $\alpha$-secretase predominantly occurs $(21,39)$. In addition, as a negative feedback, larger amounts of secreted APPs $\alpha$ might be degraded extracellularly by trypsin-like serine proteinases $(40,41)$.

The additional beneficial result of the enhanced $\alpha$-secretase cleavage of APP with increased APPs $\alpha$ production is reduced APP proteolysis in the amyloidogenic pathway, resulting in lowered levels of APPs $\beta$ and soluble amyloid peptides. This effect of ADAM10 overexpression on the amyloidogenic pathway is lower than might be expected from the high increase in APPs $\alpha$, which probably reflects the different localization of the $\alpha$ - and $\beta$-secretases: the mature active form of ADAM10 is found on the cell surface (21), whereas BACE1 is mainly active in endosomes and the trans-Golgi network (42). Although our results show only a partial inhibition of the $\beta$-secretase pathway, the reduction of soluble $A \beta$ peptides as observed in young mice is sufficient to almost completely prevent the formation of amyloid plaques in older transgenic animals.

Our observation is in line with recent studies using other APP transgenic mouse models. After overexpression of the adapter protein X11 $\alpha$ in APPswe Tg2576 transgenic mice, amyloid plaque formation was reduced, although $A \beta 42$ levels were unchanged and A $\beta 40$ levels were decreased by only about $24 \%$ (43). Overexpression of a mutant copper transporter in transgenic APP mice (TgCRND8) clearly decreased the amyloid plaque burden in the cortex and hippocampus, although the brain levels of $A \beta 40$ and $A \beta 42$ were only slightly and not significantly reduced (44).

Since purified mature ADAM10 is able to cleave soluble A $\beta$ derived peptides in vitro (21), the cleavage of soluble $A \beta$ by ADAM10 also in vivo might contribute to the strong inhibition of plaque formation. As we neither detected any additional deposits of potentially neurotoxic $\mathrm{p} 3$ peptides nor obvious morphological abnormalities in $A D A M 10 \times A P P_{[V 717 I]}$ double-transgenic mice, we conclude that stimulation of the $\alpha$-secretase pathway by ADAM10 overexpression does not contribute to neurodegeneration.

The dominant-negative mutant of ADAM10 as identified in cell culture studies (21) is shown here to inhibit the $\alpha$-secretase cleavage of APP and therefore the generation of APPs $\alpha$ in vivo. In spite of the large decrease of APPs $\alpha$, we observed only small increases in the levels of APPs $\beta$ and soluble A $\beta$ peptides. Nevertheless, doubletransgenic $A D A M 10-d n \times A P P_{[V 717 I]}$ mice developed drastically more and larger amyloid plaques than control animals, and their plaque formation started at an earlier age. This result is in line with the well-known phenomenon that very small increases of $A \beta$ peptides might finally result in amyloid deposition as diffuse and senile plaques in $\mathrm{AD}$ (1). Furthermore, our observation strengthens the hypothesis that ADAM10 in vivo also may repress amyloid plaque 
formation by cleavage of soluble $A \beta$ peptides. Inhibition of this $A \beta$ peptide cleaving activity by the dominant-negative mutant might further promote plaque formation in the AD mouse model.

In the current study, we demonstrate that ADAM10 overexpression in an $\mathrm{AD}$ animal model reverses impaired LTP and cognitive deficits early in life before plaque formation occurs. As reported (31), a neuron-specific knockout of presenilin 1 prevented amyloid plaque formation, but did not improve cognitive deficits of $A P P_{[V 717 I]}$ mice, the mouse model used in the present investigation. In our study, the beneficial effect of increased ADAM10 activity, including cognitive improvements, can most likely be attributed to the combined effects of decreased levels of toxic $A \beta$ peptides and endogenously increased amounts of neuroprotective APPs $\alpha$. Exogenously applied APPs $\alpha$ has been shown to enhance LTP in hippocampal slices (45), and in behavioral paradigms, intracerebroventricularly administered APPs $\alpha$ enhanced memory in normal and amnesic mice (46). Furthermore, a positive association between cerebrospinal fluid levels of APPs $\alpha$ and cognitive performance in rats has been reported (47).

Because neurotoxic $A \beta$ peptide levels are increased and neuroprotective APPs $\alpha$ is drastically decreased in ADAM10$d n \times A P P_{[V 717 I]}$ expressing mice, we assume that LTP should be impaired compared with $A P P_{[V 717 I]}$ mice. However, as the beneficial effect of ADAM10 overexpression was the focus of our interest, this was not analyzed.

In conclusion, the results provide strong evidence that ADAM10 acts as an efficient $\alpha$-secretase in vivo, without excluding other members of the ADAM family as $\alpha$-secretases. For treatment of $\mathrm{AD}$, activation of the $\alpha$-secretases, especially ADAM10, may be particularly promising compared with other targets, because it combines several beneficial effects: inhibition of $A \beta$ peptide production by cleavage of APP at the $\alpha$-secretase site, further reduction of the $A \beta$ level by cleavage of soluble $A \beta$ peptides, and generation of neuroprotective APPs $\alpha$. Despite intensive efforts, it has been difficult to find safe and selective $\beta$ - and $\gamma$-secretase inhibitors, mainly because of the influence of inhibitors on other substrates. Activation of proteases degrading A $\beta$ peptides, like the insulindegrading enzyme or neprilysin, represents another therapeutic approach (48). These enzymes, however, have a number of substrates, and the signaling pathways for upregulation are largely unknown at present. Cleavage of putative substrates other than APP by ADAM10 might play a role during neural development (25, $49,50)$, but probably has no effect or minor effects in adults. In our mouse model at least, we found no indications that ADAM10 affects Notch signaling in the brain of adult animals.

An increase in activity of $\alpha$-secretases, particularly ADAM10, in the brain of $\mathrm{AD}$ patients may be achieved by modulating selective signaling pathways that increase their activity or expression level. Newly developed protein kinase $\mathrm{C}$ activators with a marked loss of tumor-promoting activity that strongly enhance $\alpha$-secretase activity (51) are worth further exploring as possible therapeutics in the treatment of AD. Stimulation of receptors for neuropeptides and neurotransmitters localized in brain areas affected by AD (52) might be another valuable approach that we are examining. Muscarinic agonists increase $\alpha$-secretase activity in vitro and in vivo. In a study, AD patients treated with a selective M1 (muscarinic) agonist for 4 weeks showed a significant decrease in levels of $A \beta$ peptides in cerebrospinal fluid (53). For the discovery of novel $\alpha$-secretase activators, we developed a screening assay for libraries of small-molecular-weight compounds. Reduction of cellular cholesterol was recently shown to stimulate the generation of APPs $\alpha$ by ADAM10 and to inhibit A $\beta$ peptide formation (23). Therefore, treatment with inhibitors of cholesterol biosynthesis might be a further opportunity for an increase in $\alpha$-secretase activity and therapeutic intervention.

Finally, as another strategy, we propose the transfer of the $A D A M 10$ gene to the brain. Intravenous transfer of the tyrosine hydroxylase gene was recently successfully applied to reverse symptoms of experimental Parkinsonism (54). Using this approach, we are presently investigating in an animal model whether an increase of $\alpha$-secretase expression and activity at later stages in life could still exert such beneficial effects as shown here, since this would open approaches to a therapy of the neurodegeneration in AD.

\section{Acknowledgments}

The authors thank C. Haass and S. Sinha for antibodies, L. Zhang for technical assistance in behavioral studies, and M. Protschka for microinjection. Parts of this work have been supported by the Deutsche Forschungsgemeinschaft (to F. Fahrenholz and R. Postina), the Fritz Thyssen Foundation (to F. Fahrenholz and E. Kojro), and the Boehringer-Ingelheim Foundation (to M. Blessing). I. Dewachter and F. van Leuven were supported by the Fonds voor Wetenschappelijk Onderzoek-Vlaanderen (FWOVlaanderen) and the Katholieke Universiteit Leuven Research Fund. E. Godaux was supported by the Queen Elisabeth Fund for Medical Research, Belgium.

Received for publication December 18, 2003, and accepted in revised form February 10, 2004.

Address correspondence to: Falk Fahrenholz, Institute of Biochemistry, University of Mainz, Becherweg 30, D-55099 Mainz, Germany. Phone: 49-6131-392-5833; Fax: 49-6131-392-5348; E-mail: bio.chemie@uni-mainz.de.

Rolf Postina, Anja Schroeder, and Ilse Dewachter contributed equally to this work.
1. Selkoe, D.J. 2001. Alzheimer's disease: genes, proteins, and therapy. Physiol. Rev. 81:741-766.

2. Geula, C., et al. 1998. Aging renders the brain vulnerable to amyloid $\beta$-protein neurotoxicity. Nat. Med. 4:827-831.

3. Kayed, R., et al. 2003. Common structure of soluble amyloid oligomers implies common mechanism of pathogenesis. Science. 300:486-489.

4. Gotz, J., Chen, F., Van Dorpe, J., and Nitsch, R.M. 2001. Formation of neurofibrillary tangles in P301l tau transgenic mice induced by A $\beta 42$ fibrils. Science. 293:1491-1495.

5. Walsh, D.M., et al. 2002. Naturally secreted oligomers of amyloid $\beta$ protein potently inhibit hippocampal long-term potentiation in vivo. Nature. 416:535-539.

6. Chen, G., et al. 2000. A learning deficit related to age and $\beta$-amyloid plaques in a mouse model of Alzheimer's disease. Nature. 408:975-979.

7. Vassar, R., and Citron, M. 2000. A $\beta$-generating enzymes: recent advances in $\beta$ - and $\gamma$-secretase research. Neuron. 27:419-422.

8. Esch, F.S., et al. 1990. Cleavage of amyloid $\beta$ peptide during constitutive processing of its precursor. Science. 248:1122-1124.

9. Haass, C., Koo, E.H., Mellon, A., Hung, A.Y., and Selkoe, D.J. 1992. Targeting of cell-surface $\beta$-amyloid precursor protein to lysosomes: alternative pro- cessing into amyloid-bearing fragments. Nature. 357:500-503.

10. Sisodia, S.S. 1992. $\beta$-Amyloid precursor protein cleavage by a membrane-bound protease. Proc. Natl. Acad. Sci. U. S. A. 89:6075-6079.

11. Small, D.H., et al. 1994. A heparin-binding domain in the amyloid protein precursor of Alzheimer's disease is involved in the regulation of neurite outgrowth. J. Neurosci. 14:2117-2127.

12. Furukawa, K., et al. 1996. Increased activity-regulating and neuroprotective efficacy of $\alpha$-secretasederived secreted amyloid precursor protein conferred by a C-terminal heparin-binding domain. J. Neurochem. 67:1882-1896. 
13. Lannfelt, L., Basun, H., Wahlund, L.O., Rowe, B.A., and Wagner, S.L. 1995. Decreased $\alpha$-secretase-cleaved amyloid precursor protein as a diagnostic marker for Alzheimer's disease. Nat. Med. 1:829-832.

14. Sennvik, K., et al. 2000. Levels of $\alpha$ - and $\beta$-secretase cleaved amyloid precursor protein in the cerebrospinal fluid of Alzheimer's disease patients. Neurosci. Lett. 278:169-172.

15. Naslund, J., et al. 1994. Relative abundance of Alzheimer A $\beta$ amyloid peptide variants in Alzheimer disease and normal aging. Proc. Natl. Acad. Sci. U. S. A 91:8378-8382.

16. Gowing, E., et al. 1994. Chemical characterization of A $\beta$ 17-42 peptide, a component of diffuse amyloid deposits of Alzheimer disease. J. Biol. Chem. 269:10987-10990.

17. Higgins, L.S., Murphy, G.M., Jr., Forno, L.S., Catalano, R., and Cordell, B. 1996. P3 $\beta$-amyloid peptide has a unique and potentially pathogenic immunohistochemical profile in Alzheimer's disease brain. Am. J. Pathol. 149:585-596.

18. Wei, W., Norton, D.D., Wang, X., and Kusiak, J.W. 2002. A $\beta$ 17-42 in Alzheimer's disease activates JNK and caspase-8 leading to neuronal apoptosis. Brain. 125:2036-2043.

19. Wei, W., Wang, X., and Kusiak, J.W. 2002. Signaling events in amyloid $\beta$-peptide-induced neuronal death and insulin-like growth factor I protection. J. Biol. Chem. 277:17649-17656.

20. Koike, H., et al. 1999. Membrane-anchored metalloprotease MDC9 has an $\alpha$-secretase activity responsible for processing the amyloid precursor protein. Biochem. J. 343:371-375.

21. Lammich, S., et al. 1999. Constitutive and regulated $\alpha$-secretase cleavage of Alzheimer's amyloid precursor protein by a disintegrin metalloprotease Proc. Natl. Acad. Sci. U. S. A. 96:3922-3927.

22. Buxbaum, J.D., et al. 1998. Evidence that tumor necrosis factor $\alpha$ converting enzyme is involved in regulated $\alpha$-secretase cleavage of the Alzheimer amyloid protein precursor. J. Biol. Chem. 273:27765-27767.

23. Kojro, E., Gimpl, G., Lammich, S., Marz, W., and Fahrenholz, F. 2001. Low cholesterol stimulates the nonamyloidogenic pathway by its effect on the $\alpha$-secretase ADAM 10. Proc. Natl. Acad. Sci. U. S. A. 98:5815-5820.

24. Marcinkiewicz, M., and Seidah, N.G. 2000. Coordinated expression of $\beta$-amyloid precursor protein and the putative $\beta$-secretase BACE and $\alpha$-secretase ADAM10 in mouse and human brain. J. Neurochem. 75:2133-2143.

25. Hartmann, D., et al. 2002. The disintegrin/ metalloprotease ADAM 10 is essential for Notch signalling but not for $\alpha$-secretase activity in fibroblasts. Hum. Mol. Genet. 11:2615-2624.

26. Sinha, S., et al. 1999. Purification and cloning of amyloid precursor protein $\beta$-secretase from human brain. Nature. 402:537-540.
27. Barelli, H., et al. 1997. Characterization of new polyclonal antibodies specific for 40 and 42 amino acid-long amyloid beta peptides: their use to examine the cell biology of presenilins and the immunohistochemistry of sporadic Alzheimer's disease and cerebral amyloid angiopathy cases. Mol. Med. 3:695-707.

28. Luthi, A., et al. 1997. Endogenous serine protease inhibitor modulates epileptic activity and hippocampal long-term potentiation. J. Neurosci. 17:4688-4699.

29. Hogan, B.L.M., Beddington, R.S.P., Costantini, F., and Lacy, E. 1994. Manipulating the mouse embryo. A laboratory manual. 2nd edition. Cold Spring Harbor Laboratory Press. Cold Spring Harbor, New York, USA. 487 PP.

30. Moechars, D., et al. 1999. Early phenotypic changes in transgenic mice that overexpress different mutants of amyloid precursor protein in brain. J. Biol. Chem. 274:6483-6492.

31. Dewachter, I., et al. 2002. Neuronal deficiency of presenilin 1 inhibits amyloid plaque formation and corrects hippocampal long-term potentiation but not a cognitive defect of amyloid precursor protein [V717I] transgenic mice. J. Neurosci. 22:3445-3453.

32. Howard, L., and Glynn, P. 1995. Membrane-associated metalloproteinase recognized by characteristic cleavage of myelin basic protein: assay and isolation. Methods Enzymol. 248:388-395.

33. Paxinos, G., and Franklin, K.B.J. 2001. The mouse brain in stereotaxic coordinates. 2nd edition. Academic Press. San Diego, California, USA. 216 pp.

34. D'Hooge, R., and De Deyn, P.P. 2001. Applications of the Morris water maze in the study of learning and memory. Brain Res. Brain Res. Rev. 36:60-90.

35. Ohtsuka, T., et al. 1999. Hes1 and Hes5 as notch effectors in mammalian neuronal differentiation. EMBO J. 18:2196-2207.

36. Van Dorpe, J., et al. 2000. Prominent cerebral amyloid angiopathy in transgenic mice overexpressing the london mutant of human APP in neurons. Am. J. Pathol. 157:1283-1298.

37. Morris, R.G.M. 1984. Development of a water-maze procedure for studying spatial learning in the rat. I. Neurosci. Meth. 11:47-60.

38. Wahlsten, D., Rustay, N.R., Metten, P., and Crabbe, J.C. 2003. In search of a better mouse test. Trends Neurosci. 26:132-136.

39. Parvathy, S., Hussain, I., Karran, E.H., Turner, A.J., and Hooper, N.M. 1999. Cleavage of Alzheimer's amyloid precursor protein by $\alpha$-secretase occurs at the surface of neuronal cells. Biochemistry. 38:9728-9734.

40. Caswell, M.D., et al. 1999. The amyloid $\beta$-protein precursor of Alzheimer's disease is degraded extracellularly by a Kunitz protease inhibitor domain-sensitive trypsin-like serine protease in cultures of chick sympathetic neurons. Eur. $J$. Biochem. 266:509-516.
41. Ledesma, M.D., et al. 2000. Brain plasmin enhances APP $\alpha$-cleavage and $A \beta$ degradation and is reduced in Alzheimer's disease brains. EMBO Rep. 1:530-535.

42. Huse, J.T., Pijak, D.S., Leslie, G.J., Lee, V.M., and Doms, R.W. 2000. Maturation and endosomal targeting of $\beta$-site amyloid precursor protein-cleaving enzyme. The Alzheimer's disease $\beta$-secretase. J. Biol. Chem. 275:33729-33737.

43. Lee, J.H., et al. 2003. The neuronal adaptor protein $\mathrm{X} 11 \alpha$ reduces $\mathrm{A} \beta$ levels in the brains of Alzheimer's APPswe Tg2576 transgenic mice. J. Biol. Chem. 278:47025-47029.

44. Phinney, A.L., et al. 2003. In vivo reduction of amyloid- $\beta$ by a mutant copper transporter. Proc. Natl. Acad. Sci. U. S. A. 100:14193-14198.

45. Ishida, A., Furukawa, K., Keller, J.N., and Mattson, M.P. 1997. Secreted form of $\beta$-amyloid precursor protein shifts the frequency dependency for induction of LTD, and enhances LTP in hippocampal slices. Neuroreport. 8:2133-2137.

46. Meziane, H., et al. 1998. Memory-enhancing effects of secreted forms of the $\beta$-amyloid precursor protein in normal and amnestic mice. Proc. Natl. Acad. Sci. U. S. A. 95:12683-12688.

47. Anderson, J.J., et al. 1999. Reduced cerebrospinal fluid levels of $\alpha$-secretase-cleaved amyloid precursor protein in aged rats: correlation with spatial memory deficits. Neuroscience. 93:1409-1420.

48. Leissring, M.A., et al. 2003. Enhanced proteolysis of $\beta$-amyloid in APP transgenic mice prevents plaque formation, secondary pathology, and premature death. Neuron. 40:1087-1093.

49. Mechtersheimer, S., et al. 2001. Ectodomain shedding of $\mathrm{L} 1$ adhesion molecule promotes cell migration by autocrine binding to integrins. J. Cell Biol. 155:661-673.

50. Hattori, M., Osterfield, M., and Flanagan, J.G. 2000. Regulated cleavage of a contact-mediated axon repellent. Science. 289:1360-1365.

51. Kozikowski, A.P., et al. 2003. New amide-bearing benzolactam-based protein kinase $\mathrm{C}$ modulators induce enhanced secretion of the amyloid precursor protein metabolite sAPP $\alpha$. J. Med. Chem. 46:364-373.

52. Hooper, N.M., and Turner, A.J. 2002. The search for $\alpha$-secretase and its potential as a therapeutic approach to Alzheimer's disease. Curr. Med. Chem. 9:1107-1119.

53. Nitsch, R.M., Deng, M., Tennis, M., Schoenfeld, D., and Growdon, J.H. 2000. The selective muscarinic M1 agonist AF102B decreases levels of total A $\beta$ in cerebrospinal fluid of patients with Alzheimer's disease. Ann. Neurol. 48:913-918.

54. Zhang, Y., Calon, F., Zhu, C., Boado, R.J., and Pardridge, W.M. 2003. Intravenous nonviral gene therapy causes normalization of striatal tyrosine hydroxylase and reversal of motor impairment in experimental parkinsonism. Hum. Gene Ther. 14:1-12. 